\title{
The accessibility of topical treatment in the paranasal sinuses on operated cystic fibrosis patients assessed by scintigraphy*
}

\author{
Kasper Aanaes', Mikkel C. Alanin', Kim G. Nielsen², Mette Møller Jørgensen, \\ Johannesen ${ }^{5}$, Jann Mortensen ${ }^{4,5}$ \\ 'Department of Otorhinolaryngology, Head and Neck Surgery and Audiology, Copenhagen University Hospital, Rigshospitalet, \\ Denmark \\ 2 Danish PCD and chILD Centre, CF Centre Copenhagen, Paediatric Pulmonary Service, Department of Paediatrics and Adolescent \\ Medicine, Copenhagen University Hospital, Rigshospitalet, Denmark \\ ${ }^{3}$ Department of Clinical Microbiology, Copenhagen University Hospital, Rigshospitalet, Denmark \\ ${ }^{4}$ Department of Clinical Medicine, Faculty of Health and Medical Sciences, The Panum Institute, University of Copenhagen, \\ Denmark \\ ${ }^{5}$ Department of Clinical Physiology, Nuclear Medicine and PET, Copenhagen University Hospital, Rigshospitalet, Denmark
} Christian von Buchwald ${ }^{1}$, Niels Høiby ${ }^{3}$, Helle Krogh Johansen 3,4 Helle Hjorth

December 5, 2017

Accepted: February 8, 2018

Background: Nasal irrigations with antibiotics are used to eradicate Pseudomonas aeruginosa from the upper airways in patients with cystic fibrosis (CF) and thereby avoid lung colonisations; nevertheless, the efficacy is uncertain.

Methodology: The aim of this study was to investigate the accessibility and durability of solutions in the sinuses before and after sinus surgery. The participants irrigated their noses with radioactively marked saline and were evaluated using a dynamic SPECT/ CT scan. The preoperative and postoperative (after 30 days) examinations were compared.

Results: Twelve CF patients were included. In 10 out of the 24 scanned maxillary sinuses an improvement was seen postoperatively compared with the preoperative fluid volume. Notably, in 7 out of the 24 sinuses the mucosa was so swollen postoperatively that no fluid was detected. Ten patients had developed their frontal sinuses. We observed no fluid in the frontal or sphenoid sinuses, neither before nor after surgery. At best, a mean of $23 \%$ of the maxillary sinuses were filled with fluid; thus, all sinuses had postoperatively areas of the mucosa that did not have contact with the fluid. A mean of $76 \%$ of the initial volume was present after $30 \mathrm{~min}$ in the maxillary sinuses.

Conclusion: Fluid-depositing using nasal irrigation will not sufficiently or not at all get in contact with all the sinus mucosa despite of sinus surgery. Thus, the efficacy of topical deposition of antibiotics is presumably reduced.

Key words: cystic fibrosis, antibiotics, paranasal sinuses, eradication, nasal irrigation

\section{Introduction}

Nasal irrigations with saline reduce symptoms of sinusitis particularly in patients with cystic fibrosis (CF) and primary ciliary dyskinesia $(P C D)^{(1,2)}$, who have chronic rhinosinusitis (CRS) due to reduced mucociliary clearance. Most patients in both groups suffer from bacterial sinusitis and CRS and are in risk of pathogenic bacteria being exchanged between their sinuses and lungs; this includes Pseudomonas aeruginosa causing morbidity and mortality among patients with $\mathrm{CF}^{(3,4)}$ and is likewise a burden in

\section{$P C D^{(5)}$.}

Our group has previously shown that $P$. aeruginosa can be eradicated from the sinuses by endoscopic sinus surgery (ESS) and post-operative adjuvant treatment including nasal irrigations with antibiotics ${ }^{(6)}$. Thus, at our clinic patients with CF irrigate their nose and sinuses postoperatively with Colistimethatnatrium twice daily for three months in an attempt to transfer the principle of topical treatment using inhalations of antibiotics when these patient's lungs are infected with P. aeruginosa. 
There is a low level of evidence for use of topical antibiotic treatment in bacterial sinusitis in general; nevertheless, others and we have shown positive results in patients with $C F$ and $P C D^{(1,5-7)}$. We pioneered a management strategy for eradication of pathogenic bacteria from the paranasal sinuses of patients with CF and PCD by means of sinus surgery to mechanically remove the bacteria and create accessible sinuses for postoperative topical antibiotic treatment in combination with systemic antibiotics. Topical treatment is thought to be important due to substantially lower effects of IV-antibiotic treatments in the upper than in the lower airways as reflected in measurements of inflammatory markers ${ }^{(8)}$, because intramucosal bacteria have been described in CF patients ${ }^{(9)}$, and because mucoid $P$. aeruginosa grow as biofilm frequently concealing the mucosal lining of the sinuses being tolerant to antibiotics and the host's immune-response ${ }^{(10)}$. Most studies have used the maxillary sinuses as a model for the distribution of solutions applied by nasal irrigation devices into all sinuses; likewise, the maxillary sinuses are to our knowledge the only sinuses where extensive sinus surgery in CF is discussed in the literature ${ }^{(11,12)}$. From reviewing the literature ${ }^{(1,13)}$, the effect of topical antibiotic treatment in general is not supported by evidence and it is uncertain whether topically administered antibiotics even enters the frontal and sphenoid sinuses by nasal irrigation where pathogenic bacteria also can reside ${ }^{(11,14)}$. The duration that different antibiotic solutions should reside in the sinuses to be effective is uncertain. A contact time for more than $30 \mathrm{~min}$. has been suggested based on in vitro studies for Colistimethatnatrium ${ }^{(15,16)}$, which needs to be hydrolysed before becoming active against e.g. P. aeruginosa.

The aim of this study was to investigate the amount of solution that could be irrigated into the different sinuses before and after sinus surgery and study for how long it would stay there. The result can be used to assess the likelihood whether topical antibiotics are efficient in CF sinuses. In addition, we sought to develop a scintigraphic method to assess these questions.

\section{Methods}

\section{Inclusion}

To evaluate our current treatment strategy for bacterial sinusitis in CF we initiated a prospective intervention study where consecutive adult CF patients ( $\mathrm{N}=14$ ) were referred for the study. All patients were scheduled for sinus surgery in conformity with our previously described criteria ${ }^{(17)}$; we decided not to divide the patients according to reason for surgery (symptoms of CRS and suspicion of bacterial sinusitis). All patients received instruction by one of the authors (KA or MA) in the use of the nasal irrigation device (NielMed Sinus Rinse ${ }^{\odot}$ ) at least 14 days prior to surgery and were asked to practice daily.

\section{Irrigation and scanning}

The first scans were scheduled to the day before surgery and the second when the patient had the first out-patient visit two to three weeks after surgery. The following procedure was performed: a 2 min. static image of a ${ }^{57} \mathrm{Co}$ marker placed outside each temporal bone was obtained. A nasal irrigation was performed in upright position sequentially through both nostrils with $200 \mathrm{MBq}{ }^{99 \mathrm{~m} T c-a l b u m e n ~ c o l l o i d ~(V e n t i c o l l) ~ i n ~} 240$ $\mathrm{ml}$ isotonic saline using a sinus rinse kit. The patient was then placed in an immobilized supine position on the gamma camera couch with the head fixed with a vacuum sac. A dynamic SPECT/ CT (Single-Photon Emission Computed Tomography) was performed (one $50 \mathrm{mAs}$ low dose CT and 4 sequential SPECT each as $2 * 64$ continuous rotations of $8 \mathrm{sec}$ per projection, $128 * 128$ matrix, LEHR). Attenuation and scatter correction was performed with Astonish reconstruction (4 iterations, 16 subsets). A total of four 15 min SPECT scans where made: 1, 15, 30 and 45 min after the initial nasal irrigation. After imaging, a final nasal irrigation with saline was performed to remove radioactive fluid before the patient left the department. The scans where evaluated by two experienced clinical physiologists/radiologist (HHJ \& JM); all post-operative scans were compared with preoperative scans.

\section{Retention}

At the initial SPECT scan the total radioactivity counts in the different areas with focal uptake were measured as mean activity * volume as contoured using a threshold value of about $40 \%$ of maximum pixel count. The exact threshold value was individualized so the radioactivity volume corresponded to the volume as seen on CT. The same threshold value was used in each subjects' four dynamic SPECTS and on both study days.

To evaluate the retention over $0-60 \mathrm{~min}$ in the sinuses, the total counts at the initial scan (0-15 min) were set to $100 \%$ (initial value). The counts at the following time points $15-30 \mathrm{~min}, 30-45$ min, and 45-60 min, respectively, were compared with the initial counts and the retention was calculated by linear regression. To assess how much fluid a maxillary sinus optimally can contain, the total volume ( $\mathrm{cm}^{3}$ of air and fluid) of the postoperative maxillary sinus containing most fluid in every patient was calculated and compared with the volume of fluid the same sinus contained after 0-15 min.

\section{Ethics}

The study was approved by the local ethics committee $(\mathrm{H}-1-$ 2013-032). All patients gave written informed consent with emphasis on the additional small dose of radiation from the $\mathrm{CT}$ scans (0.4-0.5 mSv per CT scan; total $<1 \mathrm{mSv}$ ). Nevertheless, the fact that the patients received radiation made us choose a relatively small number of patients. The radiation from the saline solution was regarded as insignificant.

The company (NielMed, California) producing the irrigation bottle was neither involved in nor informed about the study. 
Table 1. Data of 12 included patients identified with age and sex.

\begin{tabular}{|c|c|c|c|c|c|c|c|c|c|c|c|c|}
\hline A & $52 M$ & $37 \mathrm{M}$ & $27 M$ & $21 M$ & $41 M$ & $20 \mathrm{M}$ & $34 \mathrm{M}$ & $32 M$ & $19 F$ & $34 F$ & $27 F$ & $24 \mathrm{~F}$ \\
\hline Pre-OP scan days & -1 & -1 & -17 & -1 & -2 & -1 & -1 & -1 & -1 & -1 & -1 & -1 \\
\hline Post-OP scan days & 21 & 14 & 19 & 37 & 22 & 69 & 20 & 14 & 14 & 57 & 16 & 56 \\
\hline Frontal sinuses pre-OP & NOpa & NOpa & - & - & N Opa & NOpa & NOpa & NOpa & NOpa & N Opa & NOpa & NOpa \\
\hline Frontal sinuses post-OP & N Opa & N Air & - & - & N Air & N Opa & N Air & NOpa & NOpa & NOpa & NOpa & N Opa \\
\hline Ethmoid/ Sphenoid pre-OP & $\mathrm{Y}$ & $\mathrm{Y}$ & $\mathrm{N}$ & $\mathrm{N}$ & $\mathrm{N}$ & $\mathrm{N}$ & $\mathrm{N}$ & Y & $\mathrm{N}$ & $\mathrm{N}$ & $\mathrm{N}$ & $\mathrm{N}$ \\
\hline Ethmoid/ Sphenoid Post-OP & Y & $\mathrm{Y}$ & $\mathrm{N}$ & $\mathrm{N}$ & Y & $\mathrm{Y}$ & $\mathrm{N}$ & $\mathrm{Y}$ & $\mathrm{Y}$ & $\mathrm{Y}$ & $\mathrm{N}$ & $\mathrm{N}$ \\
\hline
\end{tabular}

Row 1-2 show days between the scans and sinus surgery. Row 3-6 show the pre- and post-operative status of the frontal and ethmoidal/sphenodal sinuses. M: Male, F: Female; N: No, radioactivity, Y: Yes, radioactivity -:No sinuses; Opa: Opacified; Air: Aerated.

\begin{tabular}{|c|c|c|c|c|c|c|c|c|c|c|c|c|}
\hline B & $52 M$ & $37 M$ & $27 M$ & $21 \mathrm{M}$ & $41 M$ & $20 \mathrm{M}$ & $34 \mathrm{M}$ & $32 \mathrm{M}$ & $19 F$ & $34 \mathrm{~F}$ & $27 F$ & $24 F$ \\
\hline \multirow{4}{*}{$\begin{array}{l}\text { Left (LT) maxillary sinus } \\
\text { Pre-OP }\end{array}$} & 4 & 1079 & 0 & 0 & 1 & 127 & 435 & 966 & 275 & 0 & 1027 & 0 \\
\hline & 0 & 825 & 0 & 0 & 1 & 119 & 399 & 731 & 150 & 0 & 1008 & 0 \\
\hline & 0 & 588 & 0 & 0 & 0 & 141 & 435 & 537 & 179 & 0 & 735 & 0 \\
\hline & 0 & 665 & 0 & 0 & 0 & 23 & 248 & 523 & 67 & 0 & 816 & 0 \\
\hline \multirow{4}{*}{$\begin{array}{l}\text { Left (LT) maxillary sinus } \\
\text { Pre-OP }\end{array}$} & 341 & 4 & 0 & 0 & 138 & 253 & 1130 & 1252 & 848 & 525 & 631 & 0 \\
\hline & 328 & 1 & 0 & 0 & 131 & 218 & 856 & 978 & 768 & 661 & 549 & 0 \\
\hline & 167 & 0 & 0 & 0 & 216 & 289 & 884 & 1116 & 706 & 686 & 624 & 0 \\
\hline & 200 & 8 & 0 & 0 & 119 & 199 & 746 & 1225 & 526 & 573 & 499 & 0 \\
\hline \multirow{4}{*}{$\begin{array}{l}\text { Right (RT) maxillary sinus } \\
\text { Pre-OP }\end{array}$} & 258 & 1816 & 0 & 831 & 295 & 424 & 305 & 965 & 3386 & 11975 & 0 & 0 \\
\hline & 166 & 1696 & 0 & 895 & 158 & 265 & 244 & 414 & 3665 & 10508 & 0 & 0 \\
\hline & 93 & 1548 & 0 & 1077 & 105 & 222 & 365 & 546 & 3774 & 8898 & 0 & 0 \\
\hline & 59 & 1459 & 0 & 947 & 103 & 236 & 170 & 504 & 3293 & 7808 & 0 & 0 \\
\hline \multirow{4}{*}{$\begin{array}{l}\text { Right (RT) maxillary sinus } \\
\text { Post-OP }\end{array}$} & 2038 & 323 & 0 & 0 & 398 & 152 & 2895 & 540 & 1663 & 939 & 0 & 0 \\
\hline & 1054 & 158 & 0 & 0 & 218 & 80 & 2830 & 440 & 851 & 1003 & 0 & 0 \\
\hline & 585 & 160 & 0 & 0 & 175 & 134 & 2494 & 327 & 539 & 575 & 0 & 0 \\
\hline & 665 & 0 & 0 & 0 & 184 & 83 & 2318 & 409 & 256 & 634 & 0 & 0 \\
\hline $\begin{array}{l}\text { The sinus with highest } \\
\text { radioactivity }\end{array}$ & $\begin{array}{c}\text { LT } \\
\text { Post- } \\
\text { OP } \\
25 \%\end{array}$ & $\begin{array}{c}\text { RT } \\
\text { Pre-OP } \\
20 \%\end{array}$ & $0 \%$ & $\begin{array}{c}\text { RT } \\
\text { Pre-OP } \\
19 \%\end{array}$ & $\begin{array}{l}\text { RT } \\
\text { Post- } \\
\text { OP } \\
41 \%\end{array}$ & $\begin{array}{l}\text { LT } \\
\text { Post- } \\
\text { OP } \\
6 \%\end{array}$ & $\begin{array}{l}\text { RT } \\
\text { Post- } \\
\text { OP } \\
23 \%\end{array}$ & $\begin{array}{l}\text { LT } \\
\text { Post- } \\
\text { OP } \\
10 \%\end{array}$ & $\begin{array}{c}\text { LT } \\
\text { Post- } \\
\text { OP } \\
17 \%\end{array}$ & $\begin{array}{c}\text { RT } \\
\text { Pre- } \\
\text { OP } \\
20 \%\end{array}$ & $\begin{array}{c}\text { LT } \\
\text { Pre-OP } \\
51 \%\end{array}$ & $0 \%$ \\
\hline
\end{tabular}

Row $1-4$ show the radioactive counts at (0-15 min), (15-30 min), (30-45 min), and (45-60 $\mathrm{min})$, respectively. Row 5 shows the maxillary sinuses with highest percentage of area with fluid compared with total area of the sinus. M: Male, F: Female.

\section{Surgery (ESS)}

The goal for ESS in CF patients is to ventilate and drain the paranasal sinuses and to make them accessible for postoperative instrumental cleansing, inspection and irrigations. The extension of the initial surgery (e.g., exploration of the frontal or sphenoid sinuses) was decided and undertaken based on the preoperative CT scan, symptoms and perioperative findings. As a standard, we apply ESS with an uncinectomy, an anterior and posterior ethmoidectomy and a medial antrostomy, leaving a significantly enlarged maxillary ostium comprising more than half the medial maxillary wall as recommended(1). When doing revision surgery the main focus was to remove polyps and inflamed mucosa when reducing accessibility to the sinuses. However, no middle maxillary maxillectomy or Draf $2 \mathrm{~b}$ or 3 were made (extensive surgery to the maxillary and frontal sinuses, respectively.

\section{Results}

In total, 14 CF patients accepted to participate: 12 patients (5 females) were included; two were excluded: One did not show up for the examination, while one patient's scan had severe motion artefacts. The mean age was 39 years (range 19-52 years), seven patients were $\Delta \mathrm{F} 508$ homozygous and five patients were $\Delta \mathrm{F} 508$ heterozygous with an additional CF mutation. The mean number of days between the initial scan and ESS were 2 (range 1-17 days) and the mean days between ESS and the postoperative scan were 30 days (range 14-69 days) (Table 1A). By coincidence, all patients had previously had sinus surgery.

The pre- and postoperative SPECTs showed retention of radioactivity in the nasal cavity in all 12 patients, especially in the nasal vestibulum and the nasopharynx. None of the patients had aplasia or remarkable hypoplasia of their maxillary sinuses. Ten out of 12 patients had distribution to at least one of the 

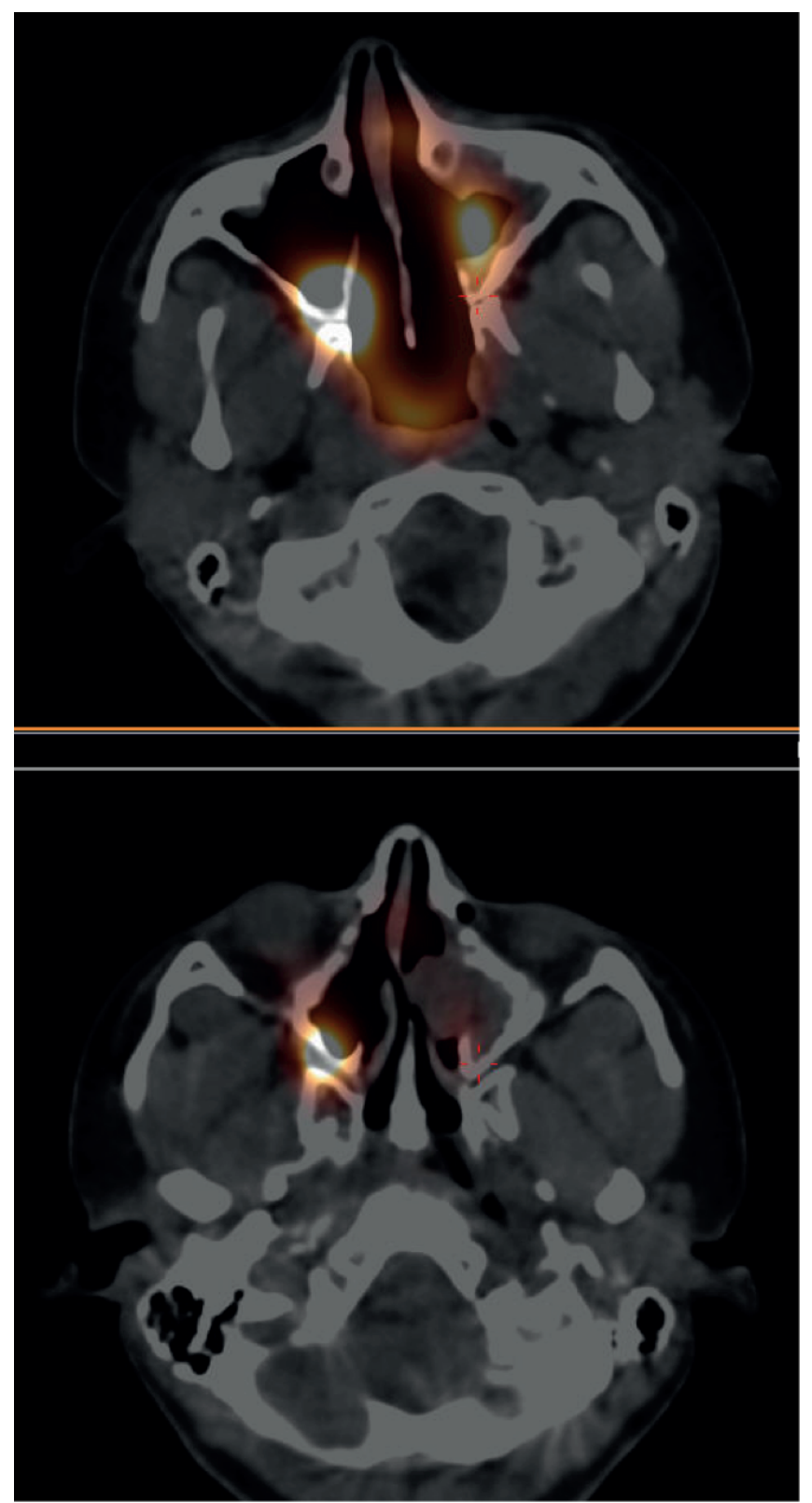

Figure 1. The postoperative SPECT/CT scan in top shows better access to the maxillary sinuses compared with the preoperative SPECT/CT scan in the bottom.

maxillary sinuses, while in two patients no retention was seen in either maxillary sinus pre- or postoperatively.

Accessibility after sinus surgery: In 10 out of the 24 scanned maxillary sinuses an improvement was seen postoperatively compared with the preoperatively fluid volume (Figure 1 \& Table 1B). Notably, in 7 out of the 24 sinuses the mucosa was so swollen postoperatively that no fluid was detected (Figure 2). There were both swollen mucosa in scans taken early (after 19 days) as well as late (after 57 days) in relation to sinus surgery opacifying the maxillary sinuses.

Ethmoidal sinuses: In eight patients, fluid could clearly be detected postoperatively lining the ethmoidal cavity at the first scan

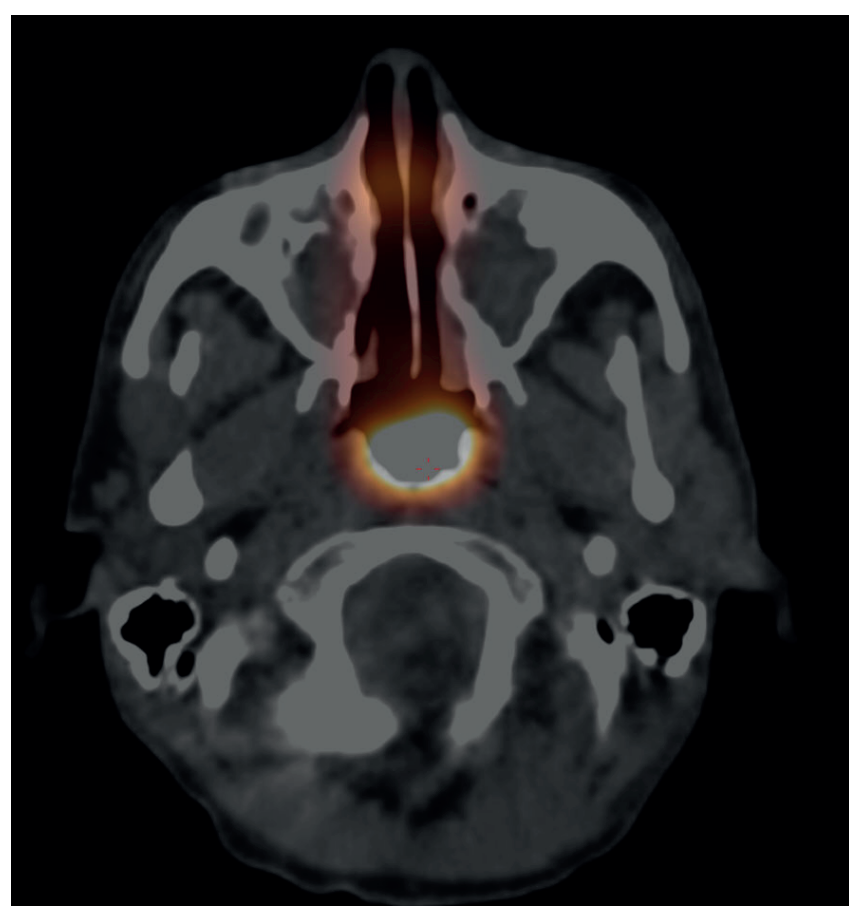

Figure 2. Postoperative SPECT/CT scan with swelling of the maxillary mucosa, thus only showing fluid in the rhinopharynx and a little in the nasal cavity.

\section{(0-15 min) (Figure 3).}

Frontal and sphenoid sinuses: A total of 10 patients had developed their frontal sinuses (all bilaterally). In none of the scans, neither preoperatively nor postoperatively, fluid could be detected in the frontal or sphenoid sinuses (Figure 3).

The amount of fluid contained in the maxillary sinuses: The patients were scanned in a steady supine position. Consequently, most volumes were positioned at the posterior maxillary wall. Two patients had at no time fluid in any of the maxillary sinuses and were excluded from these calculations. On average, the remaining ten patients had a mean of $23 \%$ (range $6-51 \%$ ) of their maxillary sinus filled with fluid; thus, postoperatively, all sinuses had areas of the mucosa that did not have contact with the fluid at the first scan (0-15 min) (Figure 4).

Duration of the fluid in the maxillary sinuses: A total of 24 sinuses were evaluated. A mean of $76 \%$ (range 23-112) of the initial volume was present after $30 \mathrm{~min}$.

\section{Discussion}

In this prospective interventional study of $12 \mathrm{CF}$ patients we found that neither pre- nor postoperative saline irrigations enter the frontal or sphenoid sinuses sufficiently. Further, the fluid was not in contact with as much sinus mucosa as we had hoped despite that the patient was immobilized after the irrigation. Surprisingly, the fluid that was in contact with the sinus mucosa at first, in general stayed there for sufficient amount of time, which could be due to the patient lying still. 


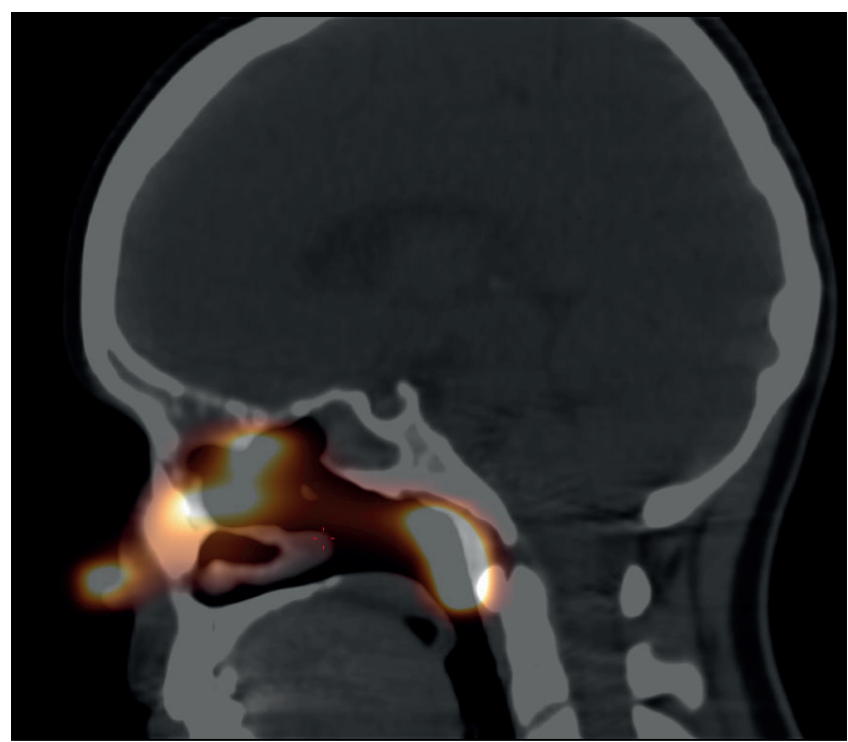

Figure 3. Postoperative SPECT/CT scan with a well accessible ethmoidal cavity but demonstrates the common picture of an opacified frontal (and sphenoidal) sinus.

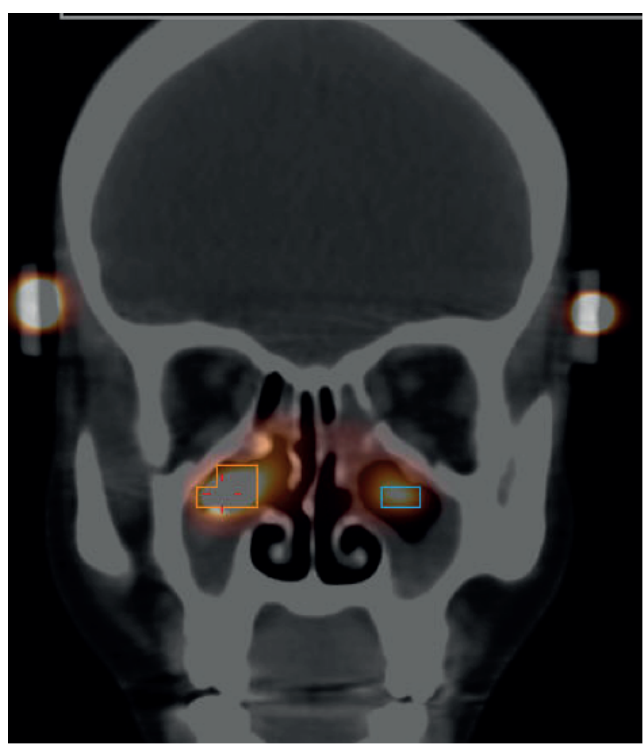

Figure 4. A SPECT/CT estimating the amount of fluid in the maxillary sinuses.
We find the consequences of these observations obvious; the optimally operative and post-operative treatment for bacterial sinusitis in CF patients has not been found, since the topical antibiotics we use are not distributed optimally. It has previously been suggested that a Draf 3 sinusotomy (a large opening to the frontal sinus) is needed for irrigations to reach the frontal sinuses ${ }^{(18)}$, thus, in upcoming surgery we will more frequently consider a Draf 3 procedure and a medial maxillectomy (larger opening to the maxillary sinuses) in CF sinus surgery - especially in revision surgery, which many CF patients need ${ }^{(19)}$. This especially applies when we do surgery with the aim of eradicating the bacteria. We are not convinced that stripping the sinus mucosa will be beneficial, but we still find it recommendable to empty the intramucosal abscesses and irrigate the sinuses during surgery.

CF patients will definitely still be recommended nasal irrigations with saline in the future, especially after ESS to remove crusts and secretion. However, the results from this study emphasize that topical administration of antibiotics should be improved. As a consequence of this study we are planning to initiate a study where topical antibiotics can be installed into the sinuses and thereby reside for a longer period of time. By optimizing the postoperative treatment for CF patients our hope is to eradicate more CF sinus bacteria and prevent chronic lung infections and thereby improve quality of life. Later, we also need to improve the treatment of the sinuses in patients with PCD, immunocomprimised patients and maybe even patients with COPD, whom all are affected with severe bacterial sinusitis ${ }^{(5,11,20)}$. By improving topical antibiotic treatment adverse systemic effects such as ototoxicity and nephrotoxicity may be reduced since use of systemic antibiotics may be reduced and viewed from a hygienic perspective it will also reduce the amount of antibiotics discharged into the environment and the spread of antibiotic resistance.

Though we find the results of this study pioneering and informative our study certainly has limitations. First of all, it should be emphazied that the first scan is made a minute after the irrigation so we can not totally rule out that a small amount of fluid transitorily entered the frontal sinuses. We chose to have the patient lying immobilized during the examination; in most cases this does not resemble daily way of life when patients perform nasal irrigation. This in mind, we have likely overestimated how much fluid and how long time it stays in the sinuses. Furthermore, due to the exposure of radiation we chose a limited number of patients; all patients had previously had sinus surgery, which may influence the outcome. We had two experienced doctors (HHJ\&JM) to evaluate the scans in consistency; optimally, we could have chosen to do the evaluation independently and blinded. By reviewing the results, we could have wished that some of the patients had been operated with a Draf 3 procedure and a medial maxillectomy why we also could have evaluated the effect of extensive surgical procedures; we would also have wished to systematically have noted the endoscopic outcomes, which might have explained why fewer than we had thought had full accessible sinuses. Nevertheless, we are convinced that the main conclusion is trustworthy and reflects the reality. Finally, though we would like to transfer our results from CF patients to other patients with bacterial sinus disease it is important to remember that CF have a unique inflammatory profile compared with others; especially, CF mucosa is often 
refractory to use of topical corticosteroids, which our patients do not systematically use.

\section{Conclusion}

In conclusion, our results show that depositing of fluid using nasal irrigation will not sufficiently achieve contact with all the sinus mucosal surfaces despite of sinus surgery; therefore, the efficacy of topical use of antibiotics is most certainly reduced. More research in best surgical practice and post-operative treatment is needed to optimize the treatment of bacterial sinusitis in CF.

\section{Acknowledgement}

The Novo Nordisk Foundation supported HKJ as a clinical research stipend.

KGN was supported by The network of COST Action BEAT-PCD: Better Evidence to Advance Therapeutic options for PCD (BM 1407).

\section{Authorship contribution}

All authors contributed in planning the study, commented on and have approved the final paper. KA and MA enrolled the patients. JM and MMJ did the scans. JM, HHJ and KA interpreted the data. KA wrote the paper.

\section{Conflict of interest}

No conflict of interest exists.

\section{References}

1. Fokkens WJ, Lund VJ, Mullol J, et al. EPOS 2012 European position paper on rhinosinusitis and nasal polyps 2012. A summary for otorhinolaryngologists.pdf. Rhinology. 2012 Mar;50(1):1-12.

2. Lucas JS, Alanin MC, Collins S, Harris A, Johansen HK, Nielsen KG, et al. Clinical care of children with primary ciliary dyskinesia. Expert Rev Respir Med. 2017 Oct 3;11(10):779-90.

3. Mainz JG, Naehrlich L, Schien M, Kading M, Schiller I, Mayr S, et al. Concordant genotype of upper and lower airways $P$ aeruginosa and $\mathrm{S}$ aureus isolates in cystic fibrosis. Thorax. 2009 Jun 1;64(6):535-40.

4. Ciofu O, Johansen HK, Aanaes K, Wassermann T, Alhede M, von Buchwald C, et al. P. aeruginosa in the paranasal sinuses and transplanted lungs have similar adaptive mutations as isolates from chronically infected CF lungs. J Cyst Fibros. 2013 Dec;12(6):729-36.

5. Alanin M. Bacteriology and treatment of infections in the upper and lower airways in patients with primary ciliary dyskinesia: adressing the paranasal sinuses. Dan Med J. 2017 May;64(5)

6. Aanaes K, von Buchwald C, Hjuler T, Skov M, Alanin M, Johansen HK. The effect of sinus surgery with intensive follow-up on pathogenic sinus bacteria in patients with cystic fibrosis. Am J Rhinol Allergy. 2013 Jan $1 ; 27(1): 1-4$

7. Lee VS, Davis GE. Culture-directed topical antibiotic treatment for chronic rhinosinusitis. Am J Rhinol Allergy. 2016 Nov 1;30(6):414-7

8. Doht F, Hentschel J, Fischer N, Lehmann T, Markert UR, Böer K, et al. Reduced effect of intravenous antibiotic treatment on sinonasal markers in pulmonary inflammation. Rhinology. 2015;53(3):249-59.
9. Kim RJT, Park L, Wood AJ, Yin T, Jain R, Douglas RG. Chronic rhinosinusitis and cystic fibrosis: the interaction between sinus bacteria and mucosal immunity. Int Forum Allergy Rhinol. 2015 May;5(5):380-5

10. Aanaes K, Eickhardt S, Johansen HK, von Buchwald C, Skov M, Høiby N, et al. Sinus biofilms in patients with cystic fibrosis: is adjusted eradication therapy needed? Eur Arch Oto-Rhino-Laryngology. 2015 Sep 9;272(9):2291-7.

11. Aanæs K. Bacterial sinusitis can be a focus for initial lung colonisation and chronic lung infection in patients with cystic fibrosis. J Cyst Fibros. 2013 Sep; 12:S1-20.

12. Virgin FW, Rowe SM, Wade MB, Gaggar A Leon KJ, Young KR, et al. Extensive surgical and comprehensive postoperative medical management for cystic fibrosis chronic rhinosinusitis. Am J Rhinol Allergy. 2012 Jan 1;26(1):70-5.

13. Varshney R, Lee JT. Current trends in topical therapies for chronic rhinosinusitis: update and literature review. Expert Opin Drug Deliv. 2017 Feb 12;14(2):257-71.

14. Grobler A, Weitzel EK, Buele A, Jardeleza C, Cheong YC, Field J, et al. Pre- and Postoperative Sinus Penetration of Nasal Irrigation. Laryngoscope. 2008 Nov;118(11):2078-81

15. Knafl D, Thalhammer F, Vossen MG. In-vitro release pharmacokinetics of amikacin, teicoplanin and polyhexanide in a platelet rich fibrin-layer (PRF) - a laboratory evaluation of a modern, autologous wound treatment. Harder J, editor. PLoS One. 2017 Jul 7;12(7):e0181090.

16. Hengzhuang W, Wu H, Ciofu O, Song Z, Hoiby N. In Vivo Pharmacokinetics/ Pharmacodynamics of Colistin and Imipenem in Pseudomonas aeruginosa Biofilm Infection. Antimicrob Agents Chemother. 2012 May 15;6(5):2683-90.
17. Aanaes K, Johansen HK, Skov M, Buchvald FF, Hjuler T, Pressler T, et al. Clinical effects of sinus surgery and adjuvant therapy in cystic fibrosis patients - Can chronic lung infections be postponed? . Vol. 51, Rhinology. 2013. p. 222-30

18. Barham HP, Ramakrishnan VR, Knisely A, Do TQ-P, Chan LS, Gunaratne DA, et al. Frontal sinus surgery and sinus distribution of nasal irrigation. Int Forum Allergy Rhinol. 2016 Mar;6(3):238-42.

19. Alanin MC, Aanaes K, Høiby N. et al.. Sinus surgery postpones chronic Gram-negative lung infection: cohort study of 106 patients with cystic fibrosis. Rhinology. 2016 Sep;54(3):206-13.

20. Håkansson K, Konge L, Thomsen SF, Backer $V$, von Buchwald C. Sinonasal inflammation in COPD: a systematic review. Eur Respir J. 2013 Nov;42(5):1402-11.

Kasper Aanæs, MD, PhD

Department of Otorhinolaryngology Head and Neck Surgery and Audio-

logy

Copenhagen University Hospital

Rigshospitalet

Blegdamsvej 9, 2100 Copenhagen

Denmark

Telephone: +45 35452071

Fax: +45 35452629

E-mail:kasper.aanaes@regionh.dk 